\title{
@creative
}

ISSN 1855-3966 (printed edn.), ISSN 1855-3974 (electronic edn.)

ARS MATHEMATICA CONTEMPORANEA 20 (2021) 37-50

https://doi.org/10.26493/1855-3974.2046.cb6

(Also available at http://amc-journal.eu)

\section{From Farey fractions to the Klein quartic and beyond*}

\author{
Ioannis Ivrissimtzis ${ }^{\dagger}$ (D) \\ Department of Computer Science, Durham University, DH1 5LE, United Kingdom \\ David Singerman (D), James Strudwick \\ Mathematical Sciences, University of Southampton, SO17 1BJ, United Kingdom
}

Received 11 July 2019, accepted 21 September 2020, published online 14 July 2021

\begin{abstract}
In a paper published in $1878 / 79$ Klein produced his famous 14-sided polygon representing the Klein quartic, his Riemann surface of genus 3 which has $\operatorname{PSL}(2,7)$ as its automorphism group. The construction and method of side pairings are fairly complicated. By considering the Farey map modulo 7 we show how to obtain a fundamental polygon for Klein's surface using arithmetic. Now the side pairings are immediate and essentially the same as in Klein's paper. We also extend his work from 7 to 11 as Klein also did in a follow-up paper of 1879 .
\end{abstract}

Keywords: Riemann surfaces, Klein quartic, regular maps, Farey tessellation, modular group, principal congruence subgroups.

Math. Subj. Class. (2020): 30F10, 20H10, 51M20

\section{Introduction}

The Klein quartic was introduced in one of Felix Klein's most famous papers, [5] of 1878/79. A slightly updated version appeared in Klein's Collected Works [7], while for a translation of this see the book The Eightfold Way, the Beauty of Klein's Quartic Curve edited by Silvio Levy [8]. This algebraic curve, whose equation is $x^{3} y+y^{3} z+z^{3} x=0$, gives the compact Riemann surface of genus 3 with 168 automorphisms, the maximum number by the Hurwitz bound.

\footnotetext{
*We thank the referees for their careful reading of this paper and their helpful suggestions.

† Coresponding author.

E-mail addresses: ioannis.ivrissimtzis@durham.ac.uk (Ioannis Ivrissimtzis), D.Singerman@soton.ac.uk (David Singerman), J.Strudwick@ soton.ac.uk (James Strudwick)
} 
Let $\mathbb{H}$ denote the upper-half complex plane and let $\mathbb{H}^{*}=\mathbb{H} \cup \mathbb{Q} \cup\{\infty\}$. Klein's surface is $\mathbb{H}^{*} / \Gamma(7)$, where $\Gamma(7)$ is the principal congruence subgroup mod 7 of the classical modular group $\Gamma=\operatorname{PSL}(2, \mathbb{Z})$. (For this concept see [4, p. 301].) Klein studies the Riemann surface of the Klein quartic by constructing his famous 14-sided fundamental region with its side identifications. See sections 11 and 12 of [5] for the construction and between pages 448 and 449 of [5], page 126 of [7], or page 320 of [8] for the figure itself.

Our approach is to construct a fundamental region for Klein's surface using the Farey tessellation $\mathcal{M}_{3}$ of $\mathbb{H}^{*}$, a triangular tessellation of $\mathbb{H}^{*}$ which we define in $\S 2$, and which was shown to be the universal triangular tessellation [10]. In $\S 3$ and $\S 4$, we study the level $n$ Farey map $\mathcal{M}_{3} / \Gamma(n)$, through the correspondence of its directed edges with the elements of $\Gamma / \Gamma(n)$ and the correspondence of its vertices with the cosets of $\Gamma_{1}(n)$ in $\Gamma$. In $\S 5$ and $\S 6$, we study the level 7 Farey map $\mathcal{M}_{3} / \Gamma(7)$. As $\mathcal{M}_{3} \subset \mathbb{H}^{*}, \mathcal{M}_{3} / \Gamma(7) \subset \mathbb{H}^{*} / \Gamma(7)$, this Farey map is embedded in the Klein surface. In a sense, we will show that this Farey map $i s$ the Klein surface.

In $\S 7$ and $\S 8$, we review Klein's original construction, computing Farey coordinates on Klein's 14-sided fundamental region and discussing the differences between the two approaches. In volume 15 of Mathematische Annalen in 1879 [6], Klein extended his work to study the surface $\mathbb{H}^{*} / \Gamma(11)$, which has $\operatorname{PSL}(2,11)$ of order 660 as its automorphism group and is somewhat more complicated. He did not draw a fundamental region for the case $n=11$ as he did for $n=7$. However we are able to draw the corresponding Farey map in $\oint 9$.

\section{The Farey map}

The vertices of the Farey map $\mathcal{M}_{3}$ are the extended rationals, i.e. $\mathbb{Q} \cup\{\infty\}$ and two rationals $\frac{a}{c}$ and $\frac{b}{d}$ are joined by an edge if and only if $a d-b c= \pm 1$. These edges are drawn as semicircles or vertical lines, perpendicular to the real axis, (i.e. hyperbolic lines). Here $\infty=\frac{1}{0}$. This map has the following properties.

(a) There is a triangle with vertices $\frac{1}{0}, \frac{1}{1}, \frac{0}{1}$, called the principal triangle.

(b) The modular group $\Gamma=\operatorname{PSL}(2, \mathbb{Z})$ acts as a group of automorphisms of $\mathcal{M}_{3}$.

(c) The general triangle has vertices $\frac{a}{c}, \frac{a+b}{c+d}, \frac{b}{d}$.

This forms a triangular tessellation of the upper half plane. Note that the triangle in (c) is just the image of the principal triangle under the Möbius transformation corresponding to the matrix $\left(\begin{array}{ll}a & b \\ c & d\end{array}\right)$.

In [10] it was shown that $\mathcal{M}_{3}$ is the universal triangular map. This means that if $\mathcal{M}$ is any triangular map on an orientable surface then $\mathcal{M}$ is the quotient of $\mathcal{M}_{3}$ by a subgroup $\Lambda$ of the modular group. A map is regular if its orientation preserving automorphism group acts transitively on its darts, (i.e. directed edges) and $\mathcal{M}_{3} / \Lambda$ is regular if and only if $\Lambda$ is a normal subgroup of $\Gamma$. The subgroup $\Lambda$ here is called a map subgroup.

(In general if $\Delta(m, n)$ is the $(2, m, n)$ triangle group, then every map of type $(m, n)$ has the form $\hat{\mathcal{M}} / M$ where $\hat{\mathcal{M}}$ is the universal map of type $(m, n)$ and $M$ is a subgroup of $\Gamma$. In our case we are thinking of the modular group $\Gamma$ as being the $(2,3, \infty)$ group. The infinity here means that we are not concerned with the vertex valencies; we just require the map to be triangular. For the general theory we refer to [3].)

We now consider the case when $\Lambda=\Gamma(n)$, the principal congruence subgroup mod $n$ of the modular group $\Gamma$. The corresponding maps are denoted by $\mathcal{M}_{3}(n)$. As $\Gamma(n)$ is a 


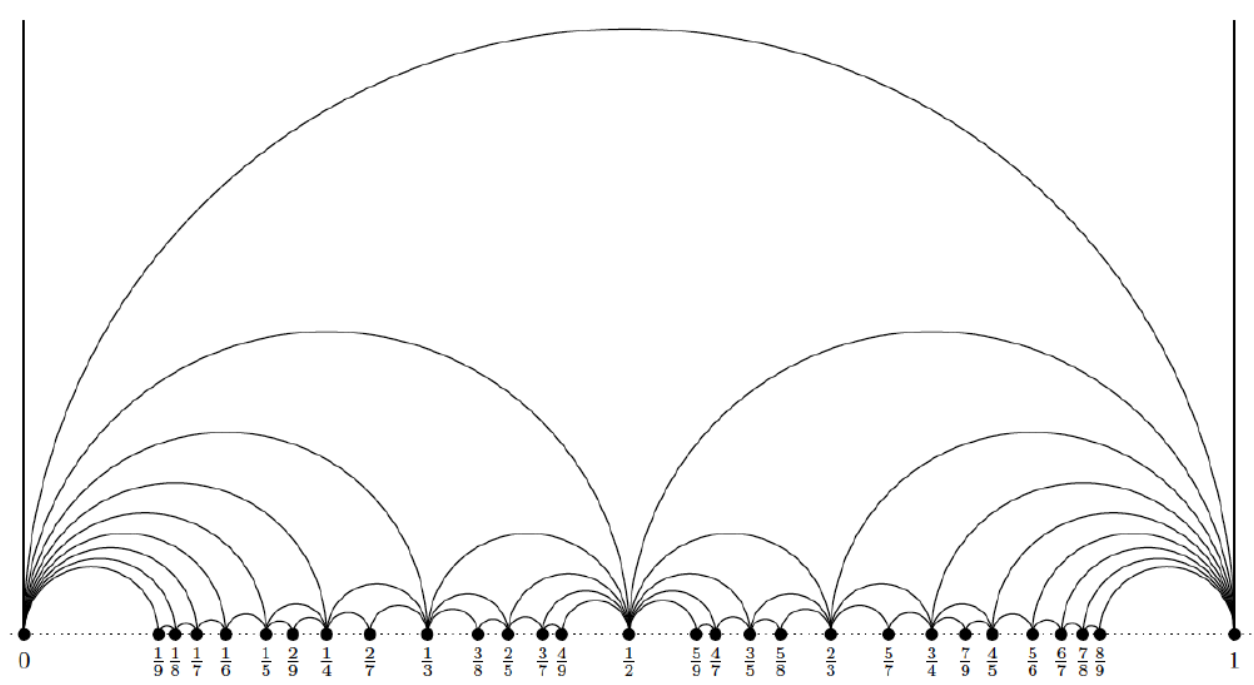

Figure 1: The Farey map, (drawn by Jan Karabaš).

normal subgroup of $\Gamma$ these maps are regular.

\section{The $\operatorname{map} \mathcal{M}_{3}(n)$}

The map $\mathcal{M}_{3}(n)$ is a regular map that lies on the Riemann surface $\mathbb{H}^{*} / \Gamma(n)$. The automorphism group of $\mathcal{M}_{3}(n)$ is $\Gamma / \Gamma(n) \cong \operatorname{PSL}\left(2, \mathbb{Z}_{n}\right)$ whose order $\mu(n)$ for $n>2$ is

$$
\mu(n)=\frac{n^{3}}{2} \prod_{p \mid n}\left(1-\frac{1}{p^{2}}\right) .
$$

The product is taken over all prime factors of $n$, see [3, Chapter 6, Exercise 6L].

Also, $\mu(2)=6$.

Now $\mu(n)$ is the number of darts of $\mathcal{M}_{3}(n)$ so the number of edges of this map is $\mu(n) / 2$, and the number of faces is equal to $\mu(n) / 3$. Note that $\frac{1}{0}$ is joined to $\frac{k}{1}$ for $k=$ $0, \ldots, n-1$ so that $\frac{1}{0}$ has valency $n$ and by regularity every vertex has valency $n$. Thus the number of vertices is equal to $\mu(n) / n$. For example, $\mu(5)=60, \mu(7)=168, \mu(11)=660$, so the numbers of vertices of $\mathcal{M}_{3}(n)$, for $n=5,7,11$, are $12,24,60$, respectively. We can now use the Euler-Poincaré formula to find the well-known formula for the genus $g(n)$ of $\mathcal{M}_{3}(n)$;

$$
g(n)=1+\frac{n^{2}}{24}(n-6) \prod_{p \mid n}\left(1-\frac{1}{p^{2}}\right) .
$$

\subsection{Farey coordinates for $\mathcal{M}_{3}(n)$}

If $(a, c, n)=1$ then the projection of $\frac{a}{c}$ from $\mathcal{M}_{3}$ to $\mathcal{M}_{3}(n)$ is denoted by $\left[\frac{a}{c}\right]$, or simply $\frac{a}{c}$ when there is no room for ambiguity, To be precise, a Farey fraction $\frac{a}{c}$ is an equivalence class of ordered pairs $(a, c) \in \mathbb{Z}_{n}^{2}$ with $(a, c, n)=1$ under the equivalence relation $(a, c) \equiv$ 
$(b, d)$ if $b=u a, d=u c \in \mathbb{Z}_{n}$ and $u= \pm 1 \in \mathbb{Z}_{n}$. This is sometimes referred to as a Farey coordinate of a vertex in $\mathcal{M}_{3}(n)$.

See $\S 4.1$ for the case $n=5$, where we give the Farey coordinates for the icosahedron.

\section{The quasi-icosahedral structure of Farey maps}

We now show that every Farey map has a quasi-icosahedral structure. Let us give some definitions from [12].

1. The (graph-theoretic) distance $\delta\left(f_{1}, f_{2}\right)$ between two vertices $f_{1}$ and $f_{2}$ of a graph is the least number of edges joining these two vertices.

2. A Farey circuit is a sequence of Farey fractions $f_{1}, f_{2}, \ldots, f_{k}$ where $f_{i}$ is joined by an edge to $f_{i+1}$ with the indices taken $\bmod k$.

3. A pole of a Farey map is any vertex with coordinates $\frac{a}{0}$.

The following theorem was proved in [12].

Theorem 4.1. Let $\frac{a}{c}, \frac{b}{d}$ be distinct vertices of $\mathcal{M}_{3}(p)$, where $p$ is prime, and let $\Delta=$ $a d-b c$. Then:

$$
\delta\left(\frac{a}{c}, \frac{b}{d}\right)= \begin{cases}1 & \text { if and only if }|\Delta|=1, \\ 2 & \text { if and only if }|\Delta| \neq 0,1, \\ 3 & \text { if and only if } \Delta=0 .\end{cases}
$$

Now let us call $\frac{1}{0}$ the north pole $N$ of $\mathcal{M}_{3}(p)$. Then by the above theorem $\delta\left(N, \frac{a}{c}\right)=1$ if and only if $c= \pm 1, \delta\left(N, \frac{a}{c}\right)=2$ if and only if $c \neq 0, \pm 1$, and $\delta\left(N, \frac{a}{c}\right)=3$ if and only if $c=0$. That is, the vertices of $\mathcal{M}_{3}(p)$ form four disjoint subsets: the north pole $N$ at $\frac{1}{0}$, a set of size $n$ consisting of vertices whose graph-theoretic distance from $N$ is 1 , another set of points at distance 2 from $N$, and other poles at distance 3 from $N$. In Theorem 4.2, we will show that these two sets are in fact circuits. As the icosahedron has this property we refer to these Farey maps as having a quasi-icosahedral structure.

(In [12] it was also shown that $\mathcal{M}_{3}(n)$ has diameter 3 for all $n \geq 5$.)

\subsection{The icosahedron}

$\mathcal{M}_{3}(5)$ is an icosahedron [12] with vertex set

$$
\left\{\frac{1}{0}, \frac{2}{0}, \frac{0}{1}, \frac{1}{1}, \frac{2}{1}, \frac{3}{1}, \frac{4}{1}, \frac{0}{2}, \frac{1}{2}, \frac{2}{2}, \frac{3}{2}, \frac{4}{2}\right\} ;
$$

see Figure 2. The north pole $N$ at $\frac{1}{0}$, there is a Farey circuit of length 5 of points whose denominator is equal to 1 and have distance 1 from $N$ and a second circuit of length 5 of points whose denominator is equal to 2 and have distance 2 from $N$. We also have the pole $\frac{2}{0}$ at distance 3 from $N$.

For a quasi-icosahedral structure on $\mathcal{M}_{3}(p)$ let $N=\frac{1}{0} \in \mathcal{M}_{3}(p)$. The circuit of points of distance 1 from $N$ is

$$
S_{1}(p)=\frac{0}{1}, \frac{1}{1}, \ldots, \frac{p-1}{1} .
$$

The set of points at distance 2 from $N$ is more complicated and we now construct it. To make the calculation clearer we start with the example $p=7$. From Theorem 4.1, we see 


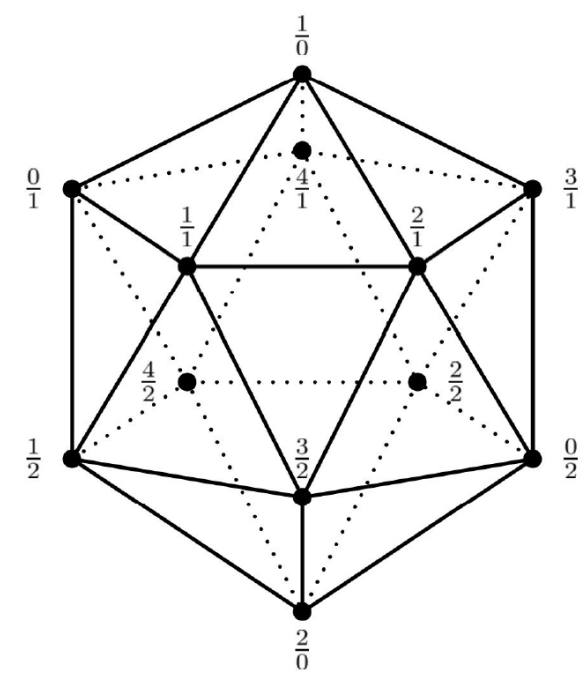

Figure 2: Drawing of $\mathcal{M}_{3}(5)$ with Farey coordinates.

that the points of distance 2 from $\frac{1}{0}$ have the form $\frac{b}{d}$ where $d= \pm 2$ or \pm 3 . Thus the points $\frac{1}{3}, \frac{1}{2}, \frac{2}{3} \in S_{2}(7)$ all have distance 2 from $N$. As the transformation $t \mapsto t+1$ fixes $N$ and preserves distance, all points in $S(7)+k$ have distance 2 from $N$, for $k=1, \ldots, 6$. Thus we find the set

$$
S_{2}(7)=\frac{1}{3}, \frac{1}{2}, \frac{2}{3}, \frac{4}{3}, \frac{3}{2}, \frac{5}{3}, \frac{0}{3}, \frac{5}{2}, \frac{1}{3}, \frac{3}{3}, \frac{0}{2}, \frac{4}{3}, \frac{6}{3}, \frac{2}{2}, \frac{0}{3}, \frac{2}{3}, \frac{4}{2}, \frac{3}{3}, \frac{5}{3}, \frac{6}{2}, \frac{6}{3}
$$

consisting of points at distance 2 from $N$, see Figure 3 . We now generalize this. Let $p \geq 5$ be a prime and let

$$
S(p)=\frac{1}{(p-1) / 2}, \frac{1}{(p-3) / 2}, \ldots, \frac{1}{3}, \frac{1}{2}, \frac{2}{3}, \ldots, \frac{(p-3) / 2}{(p-1) / 2} .
$$

Then

Theorem 4.2. The concatenation of sequences

$$
S_{2}(p)=S(S+1)(S+2) \ldots(S+p-1),
$$

where $S=S(p)$, is the Farey circuit consisting of those points of distance 2 from $N$. The length of $S_{1}(p)$ is $p$ and the length of $S_{2}(p)$ is $p(p-4)$. There are $(p-1) / 2$ poles.

Proof. We first observe that the points in $S_{2}(p)$ do have distance 2 from $N$. Indeed, the points $\frac{1}{k}$ and $\frac{m-1}{m}$ for $2 \leq k, m \leq \frac{p-1}{2}$ have distance 2 from $N=\frac{1}{0}$ as $\frac{1}{k} \leftrightarrow \frac{0}{1}$ and $\frac{m-1}{m} \leftrightarrow \frac{1}{1}$ and none of these points have distance 1 from $\frac{1}{0}$. (The symbol $\leftrightarrow$ means adjacent to.)

The transformation $t \mapsto t+1$ fixes $\frac{1}{0}$ and preserves distance so that all points in $S+k$ have distance 2 from $N=\frac{1}{0}$. We now show that $S_{2}(p)$ is a Farey circuit. Clearly there are edges between $\frac{1}{k}$ and $\frac{1}{k+1}$ for $k \geq 2$ and between $\frac{k}{k+1}$ and $\frac{k+1}{k+2}$ for $k \geq 2$. So, we only 
need to show that there is an edge between the last vertex in $S+k$ and the first vertex in $S+k+1$. The last vertex of $S+k$ is

$$
k+\frac{(p-3) / 2}{(p-1) / 2}=\frac{(p-3+k p-k) / 2}{(p-1) / 2} .
$$

The first vertex of $S+k+1$ is

$$
k+1+\frac{1}{(p-1) / 2}=\frac{(k p-k+p+1) / 2}{(p-1) / 2} .
$$

As

$$
[(p-3+k p-k) / 2][(p-1) / 2]-[(k p-k+p+1) / 2][(p-1) / 2]=-p+1,
$$

we see that the last vertex of $S+k$ is adjacent to the first vertex of $S+k+1$. Thus, $S_{2}(p)$ is a Farey circuit consisting of points of distance 2 from $\frac{1}{0}$.

Now $S_{1}(p)$ clearly has $p$ points, and the set $S(p)$ has $p-4$ points, thus $S_{2}(p)$ has $p(p-4)$ points. The poles are $\frac{1}{0}, \frac{2}{0}, \ldots$ with $\frac{k}{0}=\frac{-k}{0}$, and so the number of poles is $\frac{p-1}{2}$.

\section{Drawing $\mathcal{M}_{3}(7)$}

The map $\mathcal{M}_{3}(7)$ has 24 vertices with Farey coordinates

$$
\left\{\frac{1}{0}, \frac{2}{0}, \frac{3}{0}, \frac{0}{1}, \frac{1}{1}, \frac{2}{1}, \frac{3}{1}, \frac{4}{1}, \frac{5}{1}, \frac{6}{1}, \frac{0}{2}, \frac{1}{2}, \frac{2}{2}, \frac{3}{2}, \frac{4}{2}, \frac{5}{2}, \frac{6}{2}, \frac{0}{3}, \frac{1}{3}, \frac{2}{3}, \frac{3}{3}, \frac{4}{3}, \frac{5}{3}, \frac{6}{3}\right\} .
$$

The first circuit is $S_{1}(7)=\frac{0}{1}, \frac{1}{1}, \ldots, \frac{6}{1}$, and we draw a polygonal curve $C_{1}(7)$, surrounding $\frac{1}{0}$, containing the points of $S_{1}(7)$. We draw a bigger simple closed curve $C_{2}(7)$, also surrounding $\frac{1}{0}$, containing the points of $S_{2}(7)$. In Figure $3, C_{2}(7)$ passes through the points $\frac{1}{3}, \frac{6}{3}, \frac{6}{2}, \frac{5}{3}, \ldots$

Finally, we can draw a simple closed curve $C_{3}(7)$ exterior to both $C_{1}(7)$ and $C_{2}(7)$ which contains the poles $\frac{2}{0}$ and $\frac{3}{0}$, see the dotted line in Figure 3 . The pole $\frac{2}{0}$ is a vertex of seven triangles whose base is on the second circuit. One of these triangles is $\frac{6}{3}, \frac{2}{0}, \frac{1}{3}$ and the others are found by adding $1,2,3,4,5,6$ to these three points. For example, adding 1 to $\frac{6}{3}, \frac{2}{0}, \frac{1}{3}$ gives $\frac{2}{3}\left(=\frac{9}{3}\right), \frac{2}{0}, \frac{4}{3}$. (Adding the integer $k$ has the geometric effect of rotating $\mathcal{M}_{3}(7)$ by $\frac{2 \pi}{k}$.) The pole $\frac{3}{0}$ is a vertex of seven quadrilaterals which are unions of two Farey triangles, and also have one edge on $C_{2}(7)$. One of these is $\frac{1}{3}, \frac{5}{2}, \frac{3}{0}, \frac{1}{2}$ and we get the other six by adding $1,2,3,4,5,6$. We end up with a 42 -sided polygon pictured in Figure 3 (for now ignore the dashed curves). It is interesting that exactly the same polygon was obtained by E. Schulte and J. M. Wills in [9] by purely geometric methods.

\section{The 14-sided polygon}

We now show how to obtain a 14 -sided polygon out of the Farey map $\mathcal{M}_{3}(7)$ with the same side-pairings as the Klein surface. As $\mathcal{M}_{3}(7)$ has 42 edges and we need a 14 -sided polygon we define a new-edge to be a union of three consecutive edges which include vertices with Farey coordinates $\frac{2}{0}$ and $\frac{3}{0}$.

For example, our first new-edge goes from $\frac{2}{0}$ to $\frac{5}{3}$ to $\frac{3}{2}$ to $\frac{3}{0}$ and our second new edge goes from $\frac{3}{0}$ to $\frac{6}{2}$ to $\frac{6}{3}$ to $\frac{3}{0}$, see Figure 3 . We now replace the new-edges by dashed lines. 


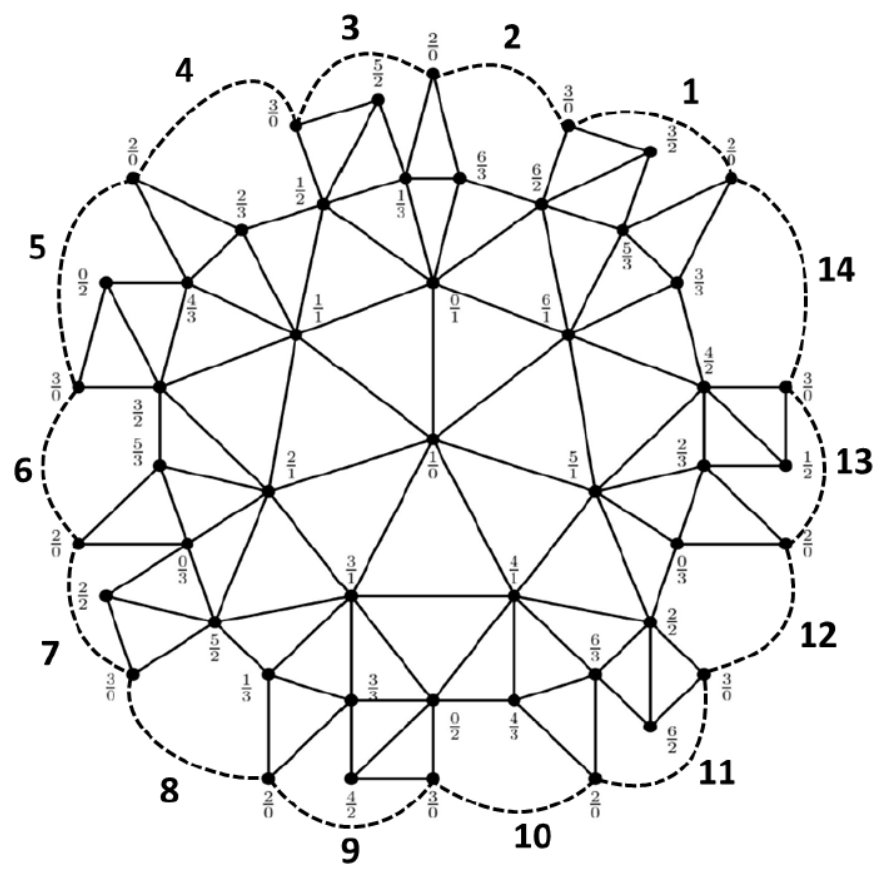

Figure 3: Drawing of $\mathcal{M}_{3}(7)$ with Farey coordinates.

In Figure 3 the dashed line labelled 1 goes from a vertex labelled $\frac{2}{0}$ to a vertex labelled $\frac{3}{0}$ surrounding the vertices $\frac{5}{3}$ and $\frac{3}{2}$ of the first new edge, and similarly the dashed line labelled 2 goes from $\frac{3}{0}$ to $\frac{2}{0}$ surrounding the vertices $\frac{6}{2}$ and $\frac{6}{3}$. Notice that the dashed lines are not part of the map $\mathcal{M}_{3}(7)$, they are just a convenient way of representing our 14sided polygon. We can associate four Farey fractions to each dashed edge. For example, associated to the dashed edge 1 we have the Farey fractions $\frac{2}{0}, \frac{5}{3}, \frac{3}{2}, \frac{3}{0}$. We pair two newedges if their associated Farey fractions are the same. For example, consider the new-edge labelled 6 in Figure 3. The associated Farey fractions are $\frac{3}{0}, \frac{3}{2}, \frac{5}{3}, \frac{2}{0}$. These are the same Farey fractions, but in reverse order as for the new-edge 1 . This means we identify the new edges 1 and 6 orientably. Similarly we get the other six identifications. Thus the identifications are

$$
1 \leftrightarrow 6,3 \leftrightarrow 8,5 \leftrightarrow 10,7 \leftrightarrow 12,9 \leftrightarrow 14,11 \leftrightarrow 2,13 \leftrightarrow 4 .
$$

This is exactly the same side-pairing as found by Klein from his 14-sided polygon which shows that our 14-sided polygon does give the Klein quartic. Our way of finding the side identifications is much more straightforward than the method used in Klein's paper, which we will summarize in $\S 8$.

\section{Farey Coordinates for the Klein map}

A regular map has type $\{m, n\}$ if every face has size $m$ and every vertex has valency $n$. (We are following [1] here and not [3] where these numbers are reversed.) Now $\mathcal{M}_{3}(n)$ is a regular map of type $\{3, n\}$ because $\frac{1}{0}$ is adjacent to $\frac{0}{1}, \ldots, \frac{n-1}{1}$. Now $\mathcal{M}_{3}(7)$ is the 
Klein map, or, in the standard notation in [1], the map $\{3,7\}_{8}$. The ' 8 ' here is the length of a Petrie polygon. (For Petrie polygons and how we find the lengths of Petrie polygons using Farey fractions see [11].)

As noted in the introduction, the Klein map $\mathcal{M}_{3}(7)$ is embedded in the Klein surface $\mathbb{H}^{*} / \Gamma(7)$. The term "Klein map" comes from the drawing on page 320 of [7], or page 120 of [8], of Klein's 14-sided polygon. After the given side identifications this does give a map on a surface of genus 3. See Figure 4 (and just ignore the Farey coordinates in this diagram for now). This is not the Klein map, for it is not regular, having vertices of different valency. It consists of 336 triangles while the Klein map $\mathcal{M}_{3}(7)$ has 56 triangles. Nevertheless, we can easily obtain the Klein map from Figure 4. The vertices of the map are the vertices of valency 14 . Before we describe the Klein map structure on this surface we show how to associate the 24 Farey fractions modulo 7 to the 24 vertices.

First, we assign the Farey coordinate $\frac{1}{0}$ to the centre point. We note that there are two circuits of seven vertices centred at $\frac{1}{0}$. We give the first circuit the Farey coordinates $\frac{0}{1}, \frac{1}{1}, \ldots, \frac{6}{1}$. If we extend the perpendicular bisector from $\frac{1}{0}$ to the hyperbolic line between $\frac{0}{1}$ and $\frac{1}{1}$ we get to another vertex of valency seven to which we assign the coordinate $\frac{0+1}{1+1}=\frac{1}{2}$. Similarly, we extend the perpendicular bisector from $\frac{1}{0}$ to the hyperbolic line between $\frac{1}{1}$ and $\frac{1}{2}$ to a vertex of valency seven which we give the Farey coordinate $\frac{3}{2}$. By continuing, we find all vertices with Farey coordinates $\frac{1}{2}, \frac{3}{2}, \frac{5}{2}, \frac{0}{2}, \frac{2}{2}, \frac{6}{2}$. Thus we have now found all vertices with Farey coordinates $\frac{x}{i}$ for $i=1,2$ and we just have to find the vertices with Farey ccordinates $\frac{x}{0}$ or $\frac{x}{3}$ which lie on the boundary of $\mathcal{K}$. After Klein's identifications shown in Figure 3, we see that the 14 corners of $\mathcal{K}$ belong to two classes, which we can label $\frac{2}{0}, \frac{3}{0}$. Between any two of these vertices there is precisely one more vertex of $\mathcal{M}_{3}(7)$. (After side identifications these vertices also have valency 14.) We can assign to them Farey coordinates of the form $\frac{x}{3}$ just by reading them off from Figure 3 . In fact, each $\frac{x}{3}$ occurs exactly twice and we can now pair sides of $\mathcal{K}$ that have exactly the same value of $x$. Again, this gives exactly the same side pairing as Klein found. We thus have two methods, in sections 7 and 8, of using Farey coordinates to get Klein's pairings just by observation.

Figure 4 gives a description of Klein's work using Farey coordinates. We see that each of the 14 sides of the boundary of $\mathcal{K}$ consists of a Farey edge and a non-Farey edge. The segment from $\frac{2}{0}$ to $\frac{x}{3}$ is a Farey edge whilst the segment from $\frac{x}{3}$ to $\frac{3}{0}$ is not a Farey edge. There is no automorphism of $\mathcal{K}$ mapping one segment to the other since all elements of $\Gamma$ map Farey edges to Farey edges. Note that by section 3, the Klein map has 24 vertices, 56 faces and 84 edges.

We now give the map structure. The vertices of the map are the points of valency 14 in Figure 4, that is, those points that have been given Farey coordinates. An edge joins points with Farey coordinates $\frac{a}{c}$ iand $\frac{b}{d}$ if and only if $a d-b c \equiv 1(\bmod 7)$. Three vertices with Farey coordinates $\frac{a}{c}, \frac{b}{d}$ and $\frac{e}{f}$ form a triangular face if and only if $e \equiv a+b(\bmod 7)$, $f \equiv c+d(\bmod 7)$. For example, there is a triangle with vertices $\frac{4}{1}, \frac{4}{3}$ and $\frac{6}{3}$ for $\frac{6}{3}$ represents the same point as $\frac{1}{4}$, for $\frac{1}{4}=\frac{-6}{-3}$.

\section{What Klein did}

Here we review Klein's original construction of his fundamental domain of the congruence subgroup $\Gamma(7)$, and show how this construction can be interpreted in terms of the Farey machinery we described above.

By the end of section 10 of [5] Klein had obtained the equation of his quartic curve and 


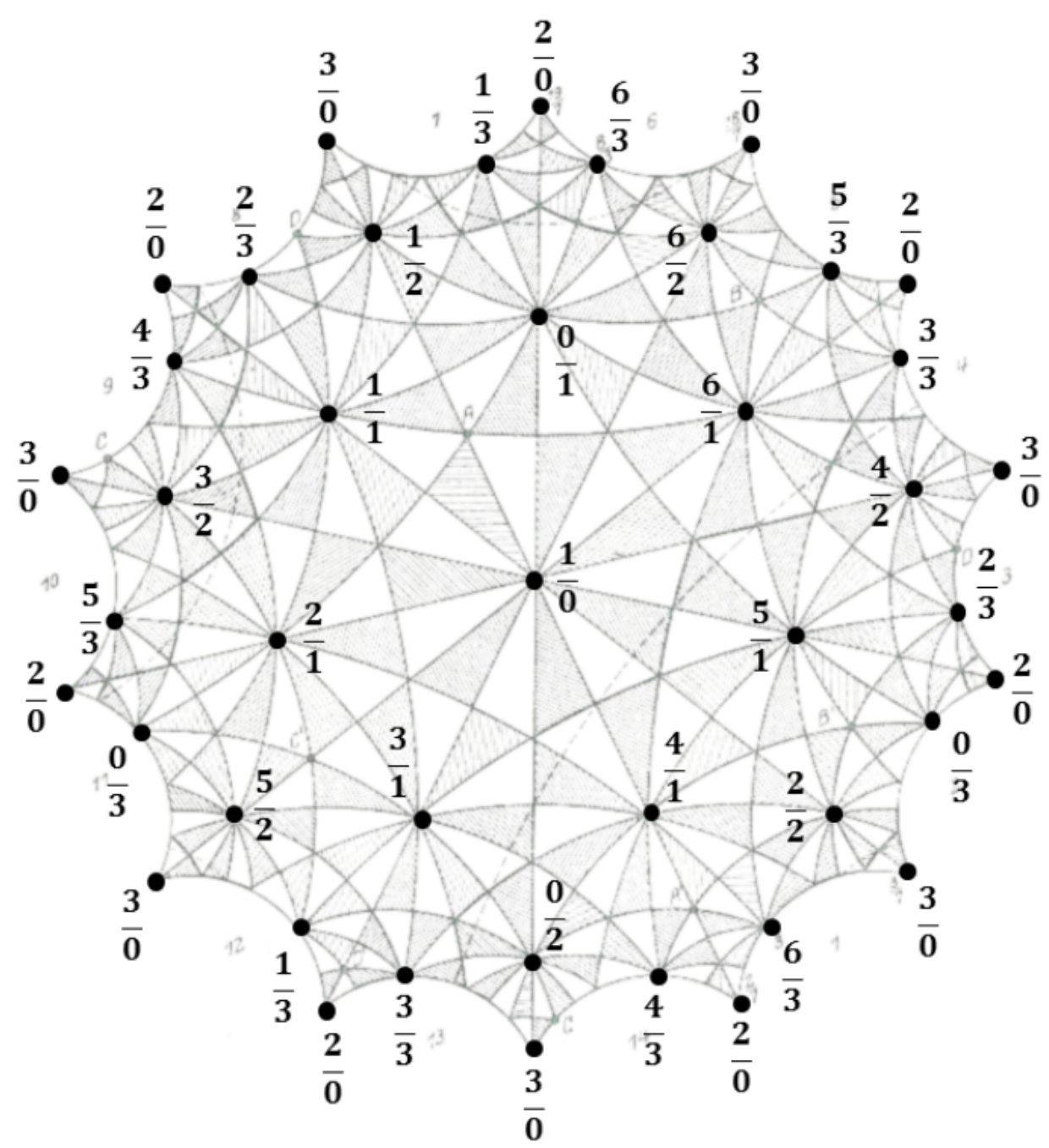

Figure 4: Farey coordinates on the Klein surface.

in section 11 he started to discuss the Riemann surface of this algebraic curve and also the corresponding map. In fact, this was one of the first publications to use maps (or in today's language dessins d'enfants) in a profound way, pointing up the deep correspondence between maps and algebraic curves. While this correspondence was not properly understood until Grothendieck's Esquisse d'un programme some 105 years later [2], we note that in an interesting anticipation of Grothendieck's programme, Figure 2 of Klein's follow-up paper [6] shows the ten planar dessins of type $(2,3,11)$ and degree 11.

Klein's quite complicated construction of his fundamental domain comes from considering fundamental regions for subgroups of indices 7 and 8 in the modular group. In section 12, he writes (in German) "In order not to make these considerations too abstract I will resort to the $\omega$-plane"; this is the upper-half plane on which the modular group acts. 
In Figure 6, he constructs a hyperbolic polygon corresponding to his 14-sided polygon describing his surface. Then, in Figure 7, he draws semicircles (hyperbolic lines) in the upper-half plane with rational vertices, which correspond to the edges of his 14-sided polygon. Now consider this polygon as being inscribed in the unit disc so the vertices all lie on the boundary circle. As the unit disc is conformally equivalent to the upper-half plane the boundary circle corresponds to the real axis and so, every point of the circle has some real coordinate. He starts with one edge (labelled 1) of his 14-sided polygon corresponding to two consecutive edges of the polygon in the upper-half plane with vertices $\frac{2}{7}, \frac{1}{3}$ and $\frac{1}{3}, \frac{3}{7}$. (As we already noted above, $\frac{2}{7}, \frac{1}{3}$ is a Farey edge while $\frac{1}{3}, \frac{3}{7}$ is not, therefore we cannot map one to the other by an element of $\Gamma$ ). A second edge (labelled 6 ) is given as the pair of consecutive edges $\frac{18}{7}, \frac{8}{3}$ and $\frac{8}{3}, \frac{19}{7}$. The Möbius transformation corresponding to the matrix

$$
\left(\begin{array}{cc}
113 & -35 \\
42 & -13
\end{array}\right)
$$

in $\Gamma(7)$ maps edge 1 (i.e. $\frac{2}{7}, \frac{1}{3}, \frac{3}{7}$ ) to edge 6 (i.e. $\frac{18}{7}, \frac{8}{3}, \frac{19}{7}$ ), and one more explicit example of edge pairing is given. He states that in total seven such matrices can be found that give all the side pairings. We feel that our technique of just using Farey coordinates is much easier.

\section{$9 \mathcal{M}_{3}(11)$}

About a year after Klein wrote his paper [5] on the quartic curve, he wrote a further paper [6] with the same title but with 'siebenter' replaced with 'elfter', i.e. 'seventh' replaced with 'eleventh'; basically, he was considering $\mathbb{H}^{*} / \Gamma(11)$. In that paper he did not draw a diagram of the fundamental region equivalent to his drawing of $\mathcal{K}$ in [5]. Here we show how to draw the Farey map $\mathcal{M}_{3}(11)$ in a similar way to how we drew $\mathcal{M}_{3}(7)$. This Farey map will be embedded in the surface $\mathbb{H}^{*} / \Gamma(11)$.

The first circuit of vertices at distance 1 from $\frac{1}{0}$ is

$$
S_{1}(11)=\frac{0}{1}, \frac{1}{1}, \ldots, \frac{10}{1}
$$

Now consider the sequence of vertices

$$
S(11)=\frac{1}{5}, \frac{1}{4}, \frac{1}{3}, \frac{1}{2}, \frac{2}{3}, \frac{3}{4}, \frac{4}{5} ;
$$

and then the second circuit is

$$
\mathcal{S}_{2}(11)=S(11)(S(11)+1) \ldots(S(11)+10) .
$$

The orientation-preserving automorphism group of $\mathcal{M}_{3}(11)$ is $\operatorname{PSL}(2,11)$ of order 660 so the Farey map $\mathcal{M}_{3}(11)$ has $660 / 2=330$ edges, $660 / 3=220$ triangles and $660 / 11=$ 60 vertices. The Farey coordinates of the vertices are $\frac{1}{0}, \frac{2}{0}, \frac{3}{0}, \frac{4}{0}, \frac{5}{0}$ and all Farey fractions of the form $\frac{r}{s}$ for $r=0$ to 10 and $s=1$ to 5 .

To draw the map we just need to find the 220 triangular faces. Because $z \mapsto z+1$ is an automorphism of $\mathcal{M}_{3}(11)$, which acts as a rotation about the centre $\frac{1}{0}$ of the map, we see that this map is divided into eleven congruent sectors each containing 220/11 = 20 triangles each. We construct one such sector $W$, shown in Figure 5, by starting from the 


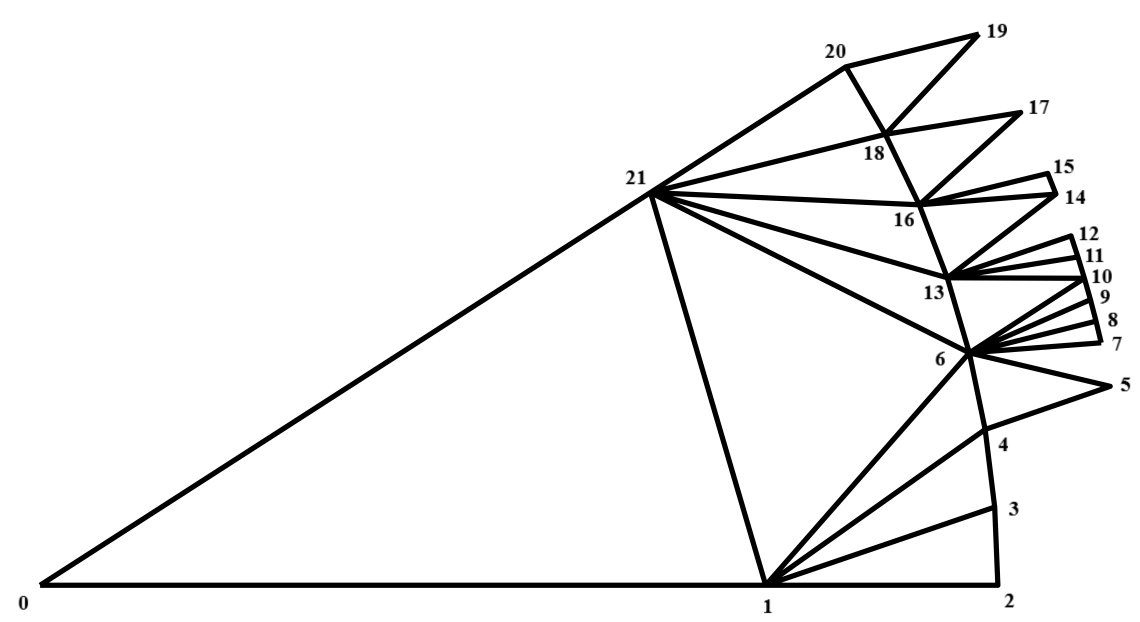

Figure 5: The sector $W$.

central triangle $\frac{1}{0}, \frac{0}{1}, \frac{1}{1}$ and adding 19 distinct triangles whose vertices lie in $S(11)$. Exactly 8 of these 19 triangles have a vertex on the first circuit $S_{1}(11)$ ( $\frac{0}{1}$ or $\frac{1}{1}$ in particular) and are uniquely determined. For the remaining 11 triangles, which either have three vertices on $S_{2}(11)$, or two vertices on $S_{2}(11)$ and a pole vertex, there are several choices satisfying the condition that they are distinct under rotation about $\frac{1}{0}$.

Figure 5 shows one such solution as the union of 20 triangles. The actual Farey coordinates are

$$
\begin{array}{rrrrrrl}
P_{0} & =\frac{1}{0} & P_{1}=\frac{0}{1} & P_{2}=\frac{1}{5} & P_{3}=\frac{1}{4} & P_{4}=\frac{1}{3} & P_{5}=\frac{2}{5} \\
P_{6}=\frac{1}{2} & P_{7}=\frac{5}{0} & P_{8}=\frac{6}{2} & P_{9}=\frac{7}{4} & P_{10}=\frac{3}{5} & P_{11}=\frac{6}{3} \\
P_{12}=\frac{4}{0} & P_{13}=\frac{2}{3} & P_{14}=\frac{6}{4} & P_{15}=\frac{3}{0} & P_{16}=\frac{3}{4} & P_{17}=\frac{4}{2} \\
P_{18}=\frac{4}{5} & P_{19}=\frac{2}{0} & P_{20}=\frac{6}{5} & P_{21}=\frac{1}{1} & &
\end{array}
$$

Each point $P_{i}$ is labelled $i$ in Figure 5 to reduce clutter.

Now let

$$
W^{*}=W \cup(W+1) \cup \cdots \cup(W+10)
$$

where $W+k$ is defined as in Section 5, that is, geometrically, is the rotation of $W$ by $\frac{2 \pi}{k}$. Then $W^{*}$ is the union of 220 triangles as required and its boundary is a polygon with $11 \times 18=198$ sides. A diagram of the map $W^{*}$ is given in Figure 6 .

Table 1 in the Appendix shows a list of the 198 boundary vertices of $W^{*}$ arranged in 11 rows. The first row corresponds to $W$ and the $k$ th row is just the first row plus $(k-1)$. We now notice that we have an orientable side pairing. For example, the first edge in row 1 going from $\frac{1}{5}$ to $\frac{1}{4}$ is paired with the edge in row 5 going from $\frac{1}{4}$ to $\frac{1}{5}$, the next edge in row 1 going from $\frac{1}{4}$ to $\frac{1}{3}$ is paired with the edge in row 8 going from $\frac{1}{3}$ to $\frac{1}{4}$. Proceeding in this way we find that all the 198 edges of the polygon are paired orientably which shows that this polygon represents an orientable surface which must be $\mathcal{M}_{3}(11)$. As the map $W^{*}$ has 60 vertices, 220 edges, and 330 triangles, by the Euler-Poincaré formula the genus of the surface is 26 . 


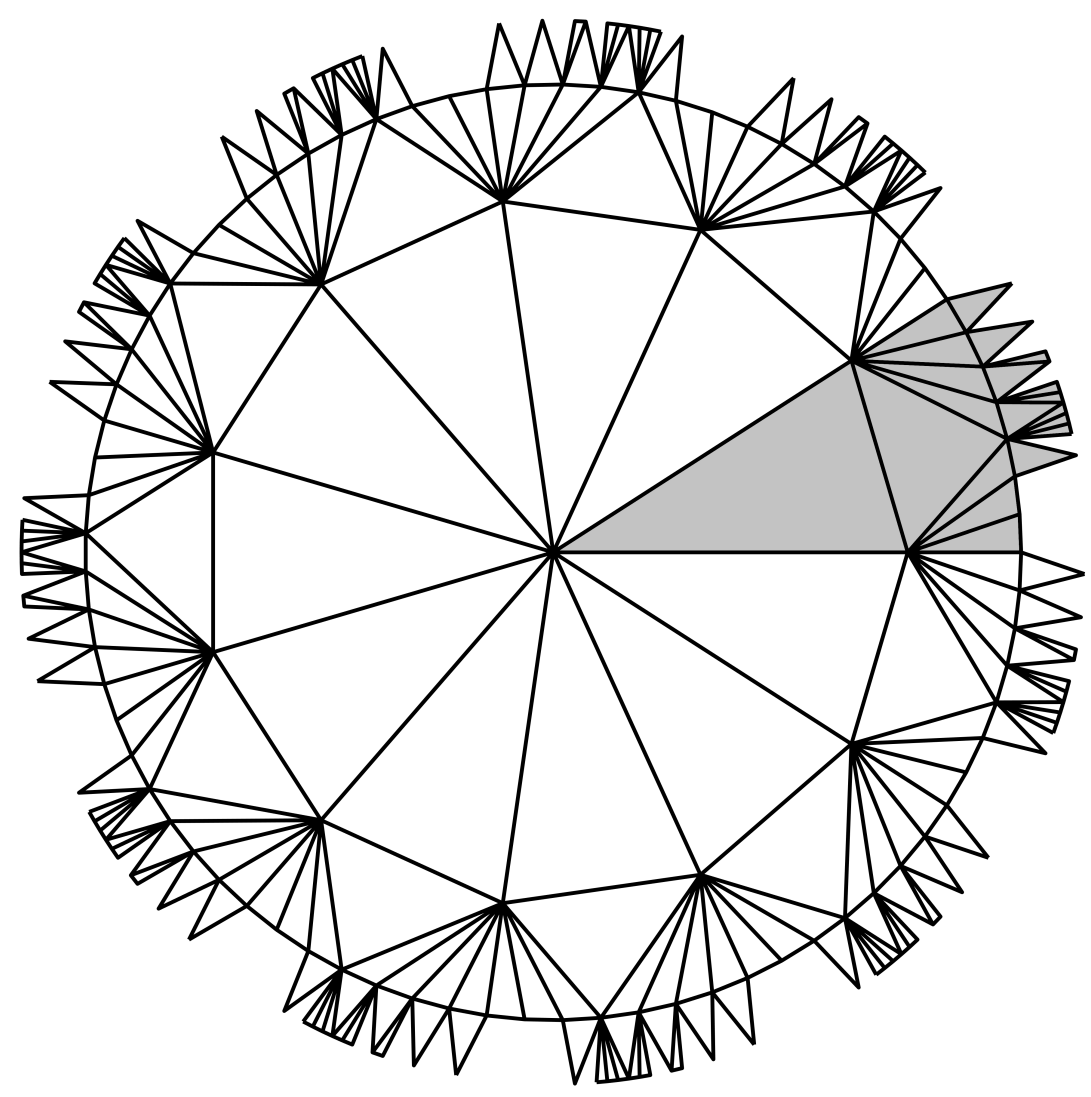

Figure 6: The map $W^{*}$.

\section{ORCID iDs}

Ioannis Ivrissimtzis (D) https://orcid.org/0000-0002-3380-1889

David Singerman (iD https://orcid.org/0000-0002-0528-5477

\section{References}

[1] H. S. M. Coxeter and W. O. J. Moser, Generators and Relations for Discrete Groups, volume 14 of Ergbnisse der Mathematik und ihre Grenzgebiete, Springer-Verlag, Berlin Heidelberg, 4th edition, 1980, doi:10.1007/978-3-662-21943-0.

[2] A. Grothendieck, Esquisse d'un programme, in: L. Schneps and P. Lochak (eds.), Geometric Galois Actions, Volume 1: Around Grothendieck's Esquisse d'un Programme, Cambridge University Press, Cambridge, volume 242 of London Mathematical Society Lecture Note Series, pp. 5-48, 1997, doi:10.1017/cbo9780511758874.003, with an English translation on pp. $243-283$.

[3] G. A. Jones and D. Singerman, Theory of maps on orientable surfaces, Proc. London Math. Soc. 37 (1978), 273-307, doi:10.1112/plms/s3-37.2.273. 
[4] G. A. Jones and D. Singerman, Complex Functions: An Algebraic and Geometric Viewpoint, Cambridge University Press, Cambridge, 1987, doi:10.1017/cbo9781139171915.

[5] F. Klein, Ueber die Transformation siebenter Ordnung der elliptischen Functionen, Math. Ann. 14 (1878), 428-471, doi:10.1007/bf01677143.

[6] F. Klein, Ueber die Transformation elfter Ordnung der elliptischen Functionen, Math. Ann. 15 (1879), 533-555, doi:10.1007/bf02086276.

[7] F. Klein, Gesammelte Mathematische Abhandlungen, Volume 3: Elliptische Funktionen, Insbesondere Modulfunktionen Hyperelliptische und Abelsche Funktionen Riemannsche Funktionentheorie und Automorphe Funktionen, Springer, Berlin Heidelberg, 1923.

[8] S. Levy, The Eightfold Way: The Beauty of Klein's Quartic Curve, volume 35 of Mathematical Sciences Research Institute Publications, Cambridge University Press, Cambridge, 1999, http://library.msri.org/books/Book35/contents.html.

[9] E. Schulte and J. M. Wills, A polyhedral realization of Felix Klein's map $\{3,7\}_{8}$ on a Riemann surface of genus 3, J. London Math. Soc. 32 (1985), 539-547, doi:10.1112/jlms/s2-32.3.539.

[10] D. Singerman, Universal tessellations, Rev. Mat. Univ. Complut. Madrid 1 (1988), 111-123, http://www. mat.ucm.es/serv/revmat/vol1-123/vol1-123h.html.

[11] D. Singerman and J. Strudwick, Petrie polygons, Fibonacci sequences and Farey maps, Ars Math. Contemp. 10 (2016), 349-357, doi:10.26493/1855-3974.864.e9b.

[12] D. Singerman and J. Strudwick, The Farey maps modulo n, Acta Math. Uni. Com. 89 (2020), 39-52, http://www.iam. fmph.uniba.sk/amuc/ojs/index.php/ amuc/article/view/913. 


\section{A Appendix}

Table 1: The boundary vertices of $W^{*}$. The last vertex of a row is repeated as the first vertex of the row below. Each row represents a sector; the first row represents sector $W$ in Figure 5. Vertices in bold belong to edges which are paired with edges in the first row $W$.

$$
\begin{aligned}
& \begin{array}{lllllllllllllllllll}
\frac{1}{5} & \frac{1}{4} & \frac{1}{3} & \frac{2}{5} & \frac{1}{2} & \frac{5}{0} & \frac{6}{2} & \frac{7}{4} & \frac{3}{5} & \frac{6}{3} & \frac{4}{0} & \frac{2}{3} & \frac{6}{4} & \frac{3}{0} & \frac{3}{4} & \frac{4}{2} & \frac{4}{5} & \frac{2}{0} & \frac{6}{5}
\end{array} \\
& \begin{array}{lllllllllllllllllll}
\frac{6}{5} & \frac{5}{4} & \frac{4}{3} & \frac{7}{5} & \frac{3}{2} & \frac{5}{0} & \frac{8}{2} & \frac{0}{4} & \frac{8}{5} & \frac{9}{3} & \frac{4}{0} & \frac{5}{3} & \frac{10}{4} & \frac{3}{0} & \frac{7}{4} & \frac{6}{2} & \frac{9}{5} & \frac{2}{0} & \frac{0}{5}
\end{array} \\
& \begin{array}{lllllllllllllllllll}
\frac{0}{5} & \frac{9}{4} & \frac{7}{3} & \frac{1}{5} & \frac{5}{2} & \frac{5}{0} & \frac{10}{2} & \frac{4}{4} & \frac{2}{5} & \frac{1}{3} & \frac{4}{0} & \frac{8}{3} & \frac{\mathbf{3}}{\mathbf{4}} & \frac{\mathbf{3}}{\mathbf{0}} & \frac{0}{4} & \frac{8}{2} & \frac{3}{5} & \frac{2}{0} & \frac{5}{5}
\end{array} \\
& \begin{array}{lllllllllllllllllll}
\frac{5}{5} & \frac{2}{4} & \frac{10}{3} & \frac{6}{5} & \frac{7}{2} & \frac{\mathbf{5}}{\mathbf{0}} & \frac{\mathbf{1}}{\mathbf{2}} & \frac{8}{4} & \frac{7}{5} & \frac{4}{3} & \frac{4}{0} & \frac{0}{3} & \frac{7}{4} & \frac{3}{0} & \frac{4}{4} & \frac{10}{2} & \frac{8}{5} & \frac{2}{0} & \frac{10}{5}
\end{array} \\
& \begin{array}{lllllllllllllllllll}
\frac{10}{5} & \frac{\mathbf{6}}{4} & \frac{2}{3} & \frac{0}{5} & \frac{9}{2} & \frac{5}{0} & \frac{3}{2} & \frac{\mathbf{1}}{\mathbf{4}} & \frac{1}{5} & \frac{7}{3} & \frac{4}{0} & \frac{3}{3} & \frac{0}{4} & \frac{3}{0} & \frac{8}{4} & \frac{1}{2} & \frac{2}{5} & \frac{2}{0} & \frac{4}{5}
\end{array} \\
& \begin{array}{lllllllllllllllllll}
\frac{4}{5} & \frac{10}{4} & \frac{5}{3} & \frac{5}{5} & \frac{0}{2} & \frac{5}{0} & \frac{5}{2} & \frac{5}{4} & \frac{6}{5} & \frac{10}{3} & \frac{4}{0} & \frac{6}{3} & \frac{4}{4} & \frac{3}{0} & \frac{1}{4} & \frac{3}{2} & \frac{7}{5} & \frac{2}{0} & \frac{9}{5}
\end{array} \\
& \begin{array}{lllllllllllllllllll}
\frac{9}{5} & \frac{3}{4} & \frac{8}{3} & \frac{10}{5} & \frac{2}{2} & \frac{5}{0} & \frac{7}{2} & \frac{9}{4} & \frac{0}{5} & \frac{2}{3} & \frac{4}{0} & \frac{9}{3} & \frac{8}{4} & \frac{3}{0} & \frac{5}{4} & \frac{5}{2} & \frac{1}{5} & \frac{2}{0} & \frac{3}{5}
\end{array} \\
& \begin{array}{llllllllllllllllllll}
\mathbf{3} & \frac{\mathbf{7}}{\mathbf{4}} & \frac{0}{3} & \frac{\mathbf{4}}{\mathbf{5}} & \frac{4}{\mathbf{2}} & \frac{5}{0} & \frac{9}{2} & \frac{2}{4} & \frac{5}{5} & \frac{5}{3} & \frac{4}{0} & \frac{\mathbf{1}}{\mathbf{3}} & \frac{\mathbf{1}}{\mathbf{4}} & \frac{3}{0} & \frac{9}{4} & \frac{7}{2} & \frac{\mathbf{6}}{\mathbf{5}} & \frac{\mathbf{2}}{\mathbf{0}} & \frac{8}{5}
\end{array} \\
& \begin{array}{lllllllllllllllllll}
\frac{8}{5} & \frac{0}{4} & \frac{3}{3} & \frac{9}{5} & \frac{6}{2} & \frac{\mathbf{5}}{\mathbf{0}} & \frac{0}{2} & \frac{6}{4} & \frac{10}{5} & \frac{8}{3} & \frac{4}{0} & \frac{4}{3} & \frac{5}{4} & \frac{3}{0} & \frac{2}{4} & \frac{9}{2} & \frac{0}{5} & \frac{2}{0} & \frac{2}{5}
\end{array} \\
& \begin{array}{lllllllllllllllllll}
\frac{2}{5} & \frac{4}{4} & \frac{\mathbf{6}}{\mathbf{3}} & \frac{\mathbf{3}}{\mathbf{5}} & \frac{8}{2} & \frac{5}{0} & \frac{2}{2} & \frac{10}{4} & \frac{4}{5} & \frac{0}{3} & \frac{4}{0} & \frac{7}{3} & \frac{9}{4} & \frac{\mathbf{3}}{\mathbf{0}} & \frac{\mathbf{6}}{\mathbf{4}} & \frac{0}{2} & \frac{5}{5} & \frac{2}{0} & \frac{7}{5}
\end{array} \\
& \begin{array}{lllllllllllllllllll}
\frac{7}{5} & \frac{8}{4} & \frac{9}{3} & \frac{8}{5} & \frac{10}{2} & \frac{5}{0} & \frac{4}{2} & \frac{3}{4} & \frac{9}{5} & \frac{3}{3} & \frac{4}{0} & \frac{10}{3} & \frac{2}{4} & \frac{3}{0} & \frac{10}{4} & \frac{2}{2} & \frac{10}{5} & \frac{2}{0} & \frac{1}{5}
\end{array}
\end{aligned}
$$

